\title{
Improvement of Drug Release Rate from Carbopol 934P Formulation
}

\author{
Takeshi Nakanishi, Fusao Kaino, and Masahiro Hayashi* \\ Department of Pharmaceutics, Faculty of Pharmaceutical Sciences, Science University of Tokyo, 12 Ichigaya, \\ Funagawara-machi, Shinjuku-ku, Tokyo 162, Japan. Received July 28, 1997; accepted October 13, 1997
}

\begin{abstract}
Carbopol 934P (CP) is a mucoadhesive polymer which has been investigated as a useful adjuvant for bioadhesive drug delivery system. However, since the drug release rate from the solid formulation of $\mathrm{CP}$ is slow, it is difficult to take advantage of the polymer's mucoadhesive property in oral administration of fast-acting drugs. In this study, we prepared freeze-dried sodium salt of $\mathrm{CP}(\mathrm{FNaCP})$ in order to improve drug release from the formulation of $\mathrm{CP}$. The drug release rate from the formulation of $\mathrm{FNaCP}$ was much faster than that of $\mathrm{CP}$ : the rate from the formulation of CP in JP XIII 1st fluid (pH 1.2) was faster than in JP XIII 2nd fluid (pH 6.8). To determine the cause of rapid drug release from $\mathrm{FNaCP}$ capsules, the change of $\mathrm{CP}$ gel properties with $\mathrm{pH}$ and ionic strength was investigated. Experimental results indicated that $\mathrm{CP}$ forms a swollen gel layer, a drug release barrier between the formulation of $\mathrm{CP}$ and the bulk release media. FNaCP was also thought to disperse rapidly in the 1 st and 2 nd fluids without formation of the swollen gel layer. In conclusion, since FNaCP improves the drug release rate from the solid CP formulation, it could be a useful adjuvant of an oral bioadhesive drug delivery system.
\end{abstract}

Key words Carbopol 934P; drug release rate; Carbopol 934P sodium salt; capsule; pH; ionic strength

Bioadhesive drug delivery is a very desirable system of oral peptide delivery. ${ }^{1}$ The main reason is that mucoadhesive polymers in the system have the possibilities of prolongation of residence time in gastrointestinal tract and increase contact time with absorbing mucosa, resulting in the enhancement of drug absorption. Carbopol 934P (CP) is a mucoadhesive polymer and has been investigated extensively by the pharmaceutical industry because of its high viscosity at low concentration and low toxicity. CP is a polyacrylic acid polymer, crosslinked with allyl sucrose. This acidic carboxyl group partially dissociates in aqueous solution, producing a flexible coil structure. Gel formation of CP depends on the electrostatic repulsion between the anionic carboxyl groups. ${ }^{2)}$ The gel behavior of $\mathrm{CP}$ has the following properties in aqueous solution: 1) the formation of gel consists of closely packed swollen particles $^{3,4)}$;2) the formation of a thick gel layer inhibits water penetration, ${ }^{5)}$ resulting in slow drug release from the CP gel. Such properties of CP are disadvantageous for oral drug delivery, in which fast action is expected from a solid formulation. Thus we prepared freeze-dried sodium salt of $\mathrm{CP}(\mathrm{FNaCP})$ to improve drug release from the $\mathrm{CP}$ formulation. The ability of $\mathrm{FNaCP}$ to improve drug release from tablets including $\mathrm{CP}$ and the effect of freeze-drying on this release from tablets were reported earlier. ${ }^{6)}$

The purpose of this study had the following two goals: 1) to compare the drug release rates between $\mathrm{FNaCP}$ and $\mathrm{CP}$ capsules; and 2) to examine differences in the properties between $\mathrm{FNaCP}$ and $\mathrm{CP}$ gels affecting these release rates.

\section{Experimental}

Materials Carbopol 934P (CP) was generously donated by BF Goodrich Co., Cleveland, OH, U.S.A. Sodium salt of CP (FNaCP) was prepared by freeze-drying an aqueous dispersion of $0.5 \% \mathrm{CP}$ which was neutralized with $10 \mathrm{M} \mathrm{NaOH}$ to $\mathrm{pH} 7.0$. Fluorescein-isothio-cyanate dextran (average molecular weight 40500, FD-40) was purchased from Sigma Chemical Co., St Louis, MO, U.S.A. Hard gelatin capsules (size 9) were purchased from Japan Elanco Company Co., Nara, Japan. All other reagents were of analytical grade or better.

Preparation of Capsules and Their Release Tests Three kinds of capsules containing FD-40 (1 mg) as a model drug with FNaCP, CP or

* To whom correspondence should be addressed. lactose $(6-8 \mathrm{mg})$ were prepared. Lactose was used as a representative excipient. Release tests of the capsules were performed in $900 \mathrm{ml}$ release media at $37^{\circ} \mathrm{C}$ using the JPXIII rotating basket method at a speed of $50 \mathrm{rpm}$. For the release media, JPXIII 1 st fluid (pH 1.2) and JPXIII 2nd fluid $(\mathrm{pH} 6.8)$ were used. The release rate of FD-40 was determined by measuring its fluorescence intensity in the release media (Hitachi spectrophotometer model F-3000).

Titration Experiments Potentiometric titrations were performed for $\mathrm{CP}$ solution by titrating $\mathrm{CP}$ solutions with standardized $0.1 \mathrm{~N} \mathrm{NaOH}$ (Horiba pH meter, F-21).

The degree of ionization, $\alpha$, was calculated by

$$
\alpha=\left\{\left[\mathrm{Na}^{+}\right]+\left[\mathrm{H}^{+}\right]-\left[\mathrm{OH}^{-}\right]\right\} /\left\{[\mathrm{PCOOH}]+\left[\mathrm{PCOO}^{-}\right]\right\}
$$

where $\left[\mathrm{Na}^{+}\right]$is the concentration of sodium ions due to the addition of $\mathrm{NaCl}$ used to adjust ionic strength, and $\left[\mathrm{H}^{+}\right]$and $\left[\mathrm{OH}^{-}\right]$are the concentrations of the hydrogen and hydroxyl ions determined from the $\mathrm{pH}$ value, respectively. The sum of $[\mathrm{PCOOH}]$ and $\left[\mathrm{PCOO}^{-}\right]$is the total polymer concentration in terms of monomeric units. The $\mathrm{p} K_{\mathrm{a}}$ value, the $\mathrm{pH}$ value at $\alpha=0.5$, was determined by linear regression analysis of the titration curve for values of $\alpha$ ranging from 0.2 to 0.8 according to the Henderson-Hasselbach relationship.

$$
\mathrm{pH}=\mathrm{p} K_{\mathrm{a}}-\ln (1-\alpha) / \alpha
$$

\section{Results}

Release Tests Figure 1 shows the FD-40 release profiles from three kinds of capsules in JPXIII 1st fluid ( $\mathrm{pH}$ 1.2). The release rate of FD-40 from the $\mathrm{FNaCP}$ capsule was more rapid than that $\mathrm{CP}$ and was equal to the control capsule including lactose. The FD-40 release profile in the 2nd fluid ( $\mathrm{pH} 6.8$ ) was similar to that in the 1st fluid (Fig. 2), so that the release from the FNaCP capsule was more rapid in both the 1st fluid and 2nd fluids. The release rate of FD-40 from the CP capsule in the 2nd fluid, in contrast, was slower than that in the 1st fluid.

During release tests, it was observed that $\mathrm{CP}$ was hydrated with both the 1st and 2nd fluids, forming a gel layer around the capsule, and this layer was thicker than in the second fluid. However, FNaCP was dispersed rapidly in both media without formation of the gel layer.

Potentiometric Titrations of CP Figure 3 showed the degree of ionization of $\mathrm{CP}$ with increase in $\mathrm{pH}$ and ionic strength $(\mu)$. Ionization of $\mathrm{CP}$ starts near $\mathrm{pH} 4$ and ends at over $\mathrm{pH}$ 8. The $\mathrm{p} K_{\mathrm{a}}$ values obtained as the $\mathrm{pH}$ values 


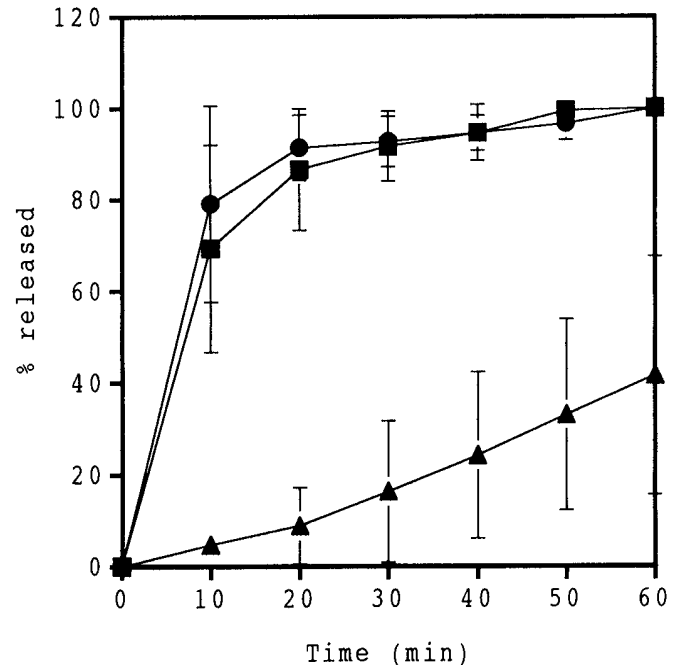

Fig. 1. In Vitro Time Profiles of FD-40 Release from Capsules in JPXIII 1st Fluid by Rotating Basket Method at $50 \mathrm{rpm}$

- FNaCP; $\boldsymbol{\Lambda}, \mathrm{CP} ; \boldsymbol{\square}$, lactose. Values represent the mean \pm S.D. $(n=4)$.

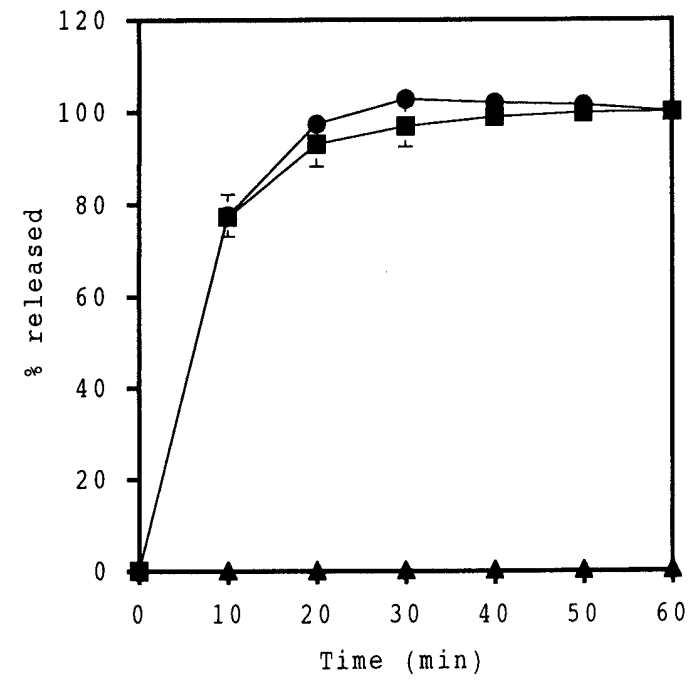

Fig. 2. In Vitro Time Profiles of FD-40 Release from Capsules in JPXIII 2nd Fluid by Rotating Basket Method at $50 \mathrm{rpm}$

- $\mathrm{FNaCP} ; \boldsymbol{\Lambda}, \mathrm{CP} ; \boldsymbol{\square}$, lactose. Values represent the mean \pm S.D. $(n=4)$.

at half ionization are listed for three $\mu$ values of $0,0.05$ and 0.1 in Table 1 . The greater the ionic strength was, the smaller was the $\mathrm{p} K_{\mathrm{a}}$ value. These results showed that ionization of $\mathrm{CP}$ depends on $\mathrm{pH}$ and $\mu$.

\section{Discussion}

As the carboxyl groups of $\mathrm{CP}$ dissociate highly in an alkaline environment (Fig. 3), electrostatic repulsion between the negatively charged carboxyl groups causes uncoiling and expansion of the molecules, resulting in swelling of the polymer and gel formation. ${ }^{2)}$ The gel is composed of closely packed swollen particles, ${ }^{3,4)}$ whose swelling increases with the increase in $\mathrm{pH}$, thus forming a thicker and more rigid gel layer. In this study, the swollen gel layer could be observed by the naked eye at the boundary between capsules of $\mathrm{CP}$ and the bulk release medium, and it became thicker with the increase in $\mathrm{pH}$. Such $\mathrm{pH}$-dependent swelling of $\mathrm{CP}$ corresponded to the fact that the release rate of FD-40 from the CP capsules

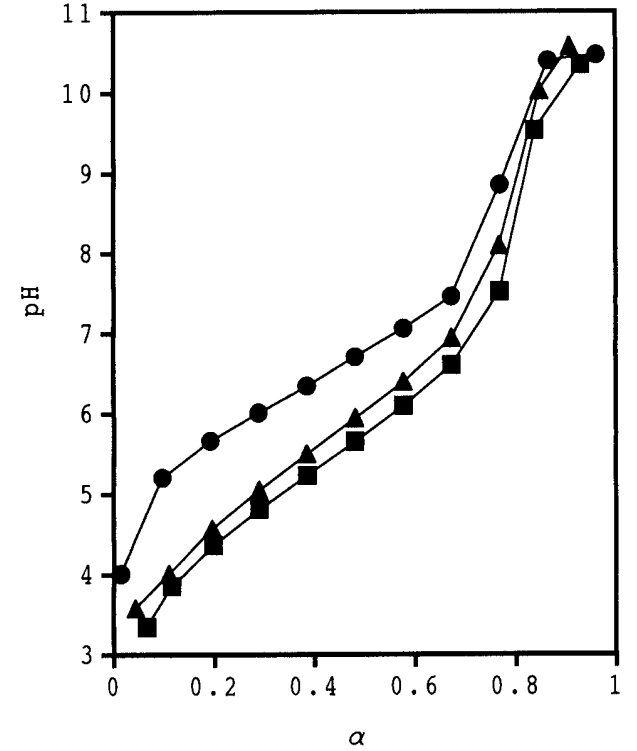

Fig. 3. Potentiometric Titration Profiles of CP $0.05 \%(w / v)$ as a Function of Ionic Strength $(\mu)$

$\alpha$ is the degree of ionization of the polymer.,$\mu=0 ; \boldsymbol{\Delta}, \mu=0.05 ; \boldsymbol{\square}, \mu=0.1$. Values represent the mean $(n=3)$.

Table 1. $\mathrm{p} K_{\mathrm{a}}$ values of $\mathrm{CP} 0.05 \%$ as a Function of Ionic Strength $(\mu)$

\begin{tabular}{lc}
\hline$\mu$ & $\mathrm{p} K_{\mathrm{a}}$ \\
\hline 0 & $6.78 \pm 0.21$ \\
0.05 & $6.18 \pm 0.01$ \\
0.1 & $5.82 \pm 0.21$ \\
\hline
\end{tabular}

The concentration of $\mathrm{CP}$ was $0.05 \%$. Ionic strength $(\mu)$ was adjusted with $\mathrm{NaCl}$. Values represent the mean \pm S.D. $(n=4)$.

was more rapid in the 1st fluid ( $\mathrm{pH} 1.2)$ than in the $2 \mathrm{nd}$ fluid (pH 6.8) (Figs. 1 and 2). Accordingly, it is suggested that the swollen gel layer restricts drug diffusion from the solid CP formulation to the bulk release media (Fig. 4a, b).

The ionization of $\mathrm{CP}$ was influenced by an increase in ionic strength of $\mathrm{CP}$ gel. With greater ionic strength, the ionization profiles of $\mathrm{CP}$ shifted to lower $\mathrm{pH}$, resulting in lower $\mathrm{p} K_{\mathrm{a}}$ (Fig. 3, Table 1). Such shift was considered to be induced by more deprotonation of the polymer in the presence of $\mathrm{Na}^{+}$ions. Screening of the carboxyl groups by $\mathrm{Na}^{+}$ions at high $\mu$ value may possibly also influence the viscosity and swelling properties of $\mathrm{CP}$ as it does other polyelectrolyte gels. ${ }^{7)}$ The ionic strength of $\mathrm{FNaCP}$ is higher than $\mathrm{CP}$ since $\mathrm{FNaCP}$ was produced by addition of $\mathrm{NaOH}$ to $\mathrm{CP}$. The release rate of FD-40 from the $\mathrm{FNaCP}$ capsules was more rapid than that of $\mathrm{CP}$ in both JPXIII 1st and 2nd solutions, independent of $\mathrm{pH}$ (Figs. 1 and 2). During the drug release from the $\mathrm{FNaCP}$ formulation, the swollen gel layer formed in $\mathrm{CP}$ was not observed (Fig. 4c). These results suggested that the ability of $\mathrm{FNaCP}$ to improve drug release from a solid formulation was attributable to the ionic strength. This difference in ionic strength between $\mathrm{CP}$ and $\mathrm{FNaCP}$ may influence the structure and the thickness of the gel layer which is a barrier to drug release from a solid formulation. Also, the difference in ionic strength between the gel and the bulk medium may determine the amount of water which 


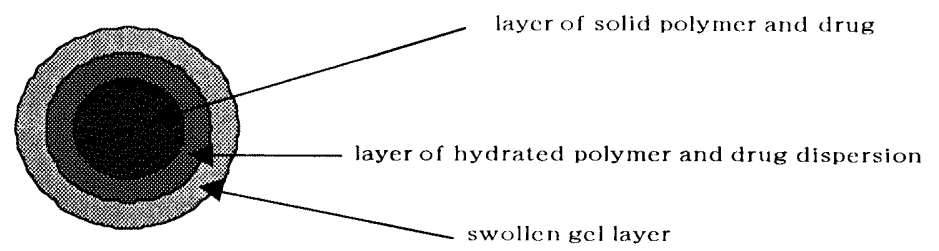

(a)

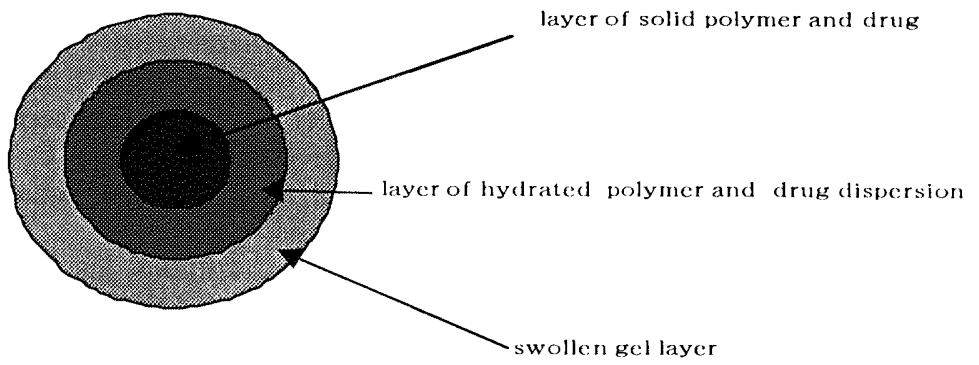

(b)

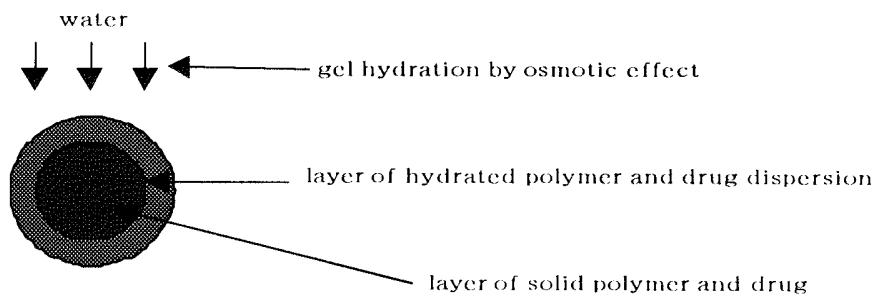

(c)

Fig. 4. Schematic Representation of Dissolution State of CP, FNaCP and Drug in Aqueous Solution of Low or High pH (a) $\mathrm{CP}$ and drug in an acidic environment. (b) $\mathrm{CP}$ and drug in an alkaline environment. (c) $\mathrm{FNaCP}$ and drug in aqueous solution.

penetrates the formulation and swells the polymer. ${ }^{8)}$ The adhesive amount of FD-40 after the perfusion of the $\mathrm{FNaCP}$ solution on small intestinal mucosa of rats was almost equal to that in CP solution (data not shown). Thus, it is concluded that the difference in release rate of FD-40 from the solid formulation of these polymers is the most important factor in enhancement of drug absorption.

In summary, the barrier to the drug release from the $\mathrm{CP}$ capsule was the swollen and thick gel layer between the capsule and the bulk release medium. For the FNaCP capsule, no such firm barrier was formed and freeze-drying in the preparation of $\mathrm{FNaCP}$ made water penetration easier, resulting in an improvement in release of the drug.
Acknowledgement The authors are grateful to Miss Kyoko Ishihara for her technical assistance.

\section{References}

1) Lueßen H. L., Lehr C.-M., Rentel C.-O., Noach A. B. J., de Boer A. G., Verhoef J. C., Junginger H. E., J. Controlled Release, 29, 329-338 (1994).

2) Dittmar C. A., Drug Cosmet. Ind., 110, 52-54, 127-128 (1972).

3) Taylor N. W., Bagley E. B., J. Appl. Polymer Sci., 18, 2747-2761 (1974).

4) Taylor N. W., Bagley E. B., J. Polymer Sci., 13, 1133-1 144 (1975)

5) French D. L., Himmelstein K. J., Mauger J. W., J. Controlled Release, 37, 281-289 (1995).

6) Kaiho F., Lueßen H. L., Lehr C.-M., Verhoef J. C., Junginger H. E., S.T.P. Pharma Sci., 6, 385-389 (1996)

7) Ünlu N., Ludwig A., Ootenghem M. V., Hincal A. A., Pharm. Acta Helv., 67, 5-10 (1992).

8) Hooper H. H., Baker J. P., Blanch H. W., Prausnitz J. M., Macromolecules, 23, 1096-1104 (1990). 